\title{
Restoration of Ovarian Function After Orthotopic Ovarian Autotransplant and Follow-Up: A Case Report
}

\author{
Renuka Devi Rajkumaralala, b, Hemashree Rajesha, Su Ling Yu ${ }^{a}$
}

\begin{abstract}
A 40-year-old married nulliparous female diagnosed with breast cancer was referred for fertility preservation before initiation of chemotherapy. Various options were discussed and she decided for ovarian tissue cryopreservation to avoid delay in treatment for cancer. She underwent laparoscopic left oophorectomy and the retrieved ovarian cortex was cryopreserved in liquid nitrogen. Two months after initiation of chemotherapy, her menses stopped and she started experiencing hot flushes leading to a diagnosis of premature menopause based on her hormone levels. Two years later after being certified free of malignancy, she underwent orthotopic transplant of the cryopreserved ovarian tissue into the other ovary and ovarian fossa. She resumed menstruation 3 months later and her hormone analysis showed a significant drop in follicle-stimulating hormone levels and luteinizing hormone levels and rise in estrogen levels. At this point of time, she lost interest in fertility and declined in vitro fertilization. On regular follow-up to assess her ovarian function, she reported regular monthly menses for 2 years which then started becoming irregular and less frequent. During her recent review 4 years after the transplant, her menses is less frequent once in $2-3$ months with light flow but free of menopausal symptoms. Ovarian cryopreservation and autotransplant is usually done to restore fertility in women undergoing gonadotoxic treatment. This case shows that this procedure can also be considered for women who wish to restore hormonal function thereby alleviating menopausal symptoms and improving bone and cardiovascular health even though such benefits are yet to be proven scientifically. Careful selection of cases and adequate counselling regarding the benefits, risks, cost of the procedure, uncertainty of duration of ovarian function and multidisciplinary approach are important to optimize outcome in such patients.
\end{abstract}

Keywords: Ovarian autotransplant; Fertility preservation; Premature menopause; Gonadotoxic chemotherapy

Manuscript accepted for publication November 24, 2016

aDepartment of Obstetrics and Gynaecology, Singapore General Hospital, Singapore

${ }^{b}$ Corresponding Author: Renuka Devi Rajkumaralal, Department of Obstetrics and Gynaecology Singapore General Hospital, Outram Road, 169608, Singapore. Email: renuka.rajkumaralal@sgh.com.sg

doi: https://doi.org/10.14740/jmc2701w

\section{Introduction}

The first live birth following orthotopic autotransplant of cryopreserved ovarian tissue was reported more than a decade ago which offered incredible promise in restoring fertility for women with malignancies who underwent gonadotoxic chemotherapy $[1,2]$. This breakthrough technique has resulted in birth of more than 60 live births worldwide with increasing number of women opting for fertility preservation when posed with risk of losing fertility due to exposure to gonadotoxic chemotherapy drugs during treatment for their malignancies [3]. In addition to preservation of fertility, return of hormonal function following ovarian autotransplant helps improve quality of life by providing a beneficial effect on symptoms like hot flushes, decreased libido, and sleep disorders and may possibly have an effect on prevention of osteoporosis, cardiovascular diseases and memory impairment $[1,4]$. This case report is about a woman who had cryopreservation of ovarian tissue for fertility preservation before initiating chemotherapy for breast cancer, underwent an orthotopic autotransplant of her cryopreserved ovarian tissue and eventually lost interest in fertility but still benefited from restoration of her ovarian hormonal function which lasted for a few years.

\section{Case Report}

A 40-year-old married nulliparous female was referred to the Centre of Assisted Reproduction, Singapore General Hospital in January 2010 for fertility preservation soon after she underwent surgery for stage 2 cancer of the left breast. Histology of the excised lesion was reported as invasive ductal carcinoma grade 3 which was negative for estrogen and progesterone receptors but positive for HER2 without involvement of lymph nodes. She was scheduled to undergo chemotherapy a few days later and various options available for fertility preservation were discussed with her, out of which she opted to undergo ovarian tissue cryopreservation after a detailed counselling regarding the risks, benefits, cost and efficacy of the procedure. She underwent laparoscopic left oophorectomy and the retrieved ovary was immediately transported to the in vitro fertilization (IVF) laboratory for further processing before cryopreservation. In the laboratory, the ovarian cortex was carefully separated from the medulla and cut into 11 small strips 
and stored in liquid nitrogen for future use. Shortly, the patient was subjected to adjuvant chemotherapy and radiotherapy for breast cancer with AC-TH regime - cyclophosphamide and doxorubicin 3 weekly for four cycles followed by paclitaxel weekly for 12 cycles and herceptin weekly for 1 year which she completed successfully.

Two months after the start of chemotherapy, she ceased having menses and also started to experience symptoms like hot flushes. Her hormone profile was assessed which showed a serum follicle-stimulating hormone (FSH) level of $164 \mathrm{IU} / \mathrm{L}$ and a luteinizing hormone (LH) level of $76 \mathrm{IU} / \mathrm{L}$ and a diagnosis of premature ovarian failure due to chemotherapy was made. Her hot flushes were kept under control by herbal supplements like black cohosh. Two years after the ovarian tissue collection and cryopreservation, she presented to the Centre of Assisted Reproduction requesting for ovarian autotransplant after being certified by the breast surgeon to be in remission with no evidence of recurrence of malignancy. Her hormone profile was tested again which was in the postmenopausal range. Histological analysis of one strip of the frozen ovarian tissue was done to rule out malignancy before scheduling her for orthotopic ovarian tissue autotransplant in August 2012.

On the day of surgery, the cryopreserved ovarian tissue was thawed $1 \mathrm{~h}$ before start of surgery and transported to the operating theater. Under general anesthesia, through a minilaparotomy incision three longitudinal incisions were made on the right ovary, seven pieces of the cryopreserved ovarian tissue were placed in the incision site and closure was done with 3-0 Vicryl whereas the remaining three pieces of the ovarian tissue were transplanted into the right ovarian fossa. The patient had an uneventful postoperative period and was discharged home on the third postoperative day. She resumed normal menses 3 months after surgery and her hormone analysis showed a significant drop in FSH and LH measuring 14.7 and $17.6 \mathrm{IU} / \mathrm{L}$, respectively and a rise in E2 levels measuring 1,151 pmol/L and antiMullerian hormone measured $2.6 \mathrm{ng} / \mathrm{mL}$. She was not keen for fertility at that point of time and declined IVF. On regular follow-up for assessment of ovarian function, she reported regular monthly menses for 2 years initially after which her menses started becoming irregular and less frequent. During a recent review 4 years after surgery, patient still has menses once in 2 3 months with light flow but is free of menopausal symptoms.

\section{Discussion}

With latest advances in the field of medicine leading to increased life expectancy of cancer survivors, there is a tremendous shift in focus and priority on fertility and quality of life for young cancer survivors who are at risk of losing their fertility potential due to gonadotoxic chemotherapy drugs like alkylating agents or radiotherapy used for malignancy treatment [5-9]. In addition to those with malignancies, women with certain benign conditions such as hematologic and autoimmune diseases also undergo treatment with gonadotoxic drugs or radiation which may put their fertility at stake [6-8, 10]. For these women who desire fertility preservation, one of the options available is removal of one ovary for cryopreserva- tion before starting chemotherapy or radiotherapy followed by autotransplant at orthotopic (other ovary or pelvic peritoneum) or heterotopic sites (subcutaneous tissue of abdominal wall, forearm and chest wall) few years later after they are cured of cancer $[2,11,12]$. The main advantages of this procedure over other options like oocyte or embryo cryopreservation are avoidance of delay in initiation of treatment for malignancy, storage of large number of ovarian follicles in a single procedure, it can be offered to prepubertal girls, does not require a partner and most importantly the possibility of having natural conception after orthotopic autotransplant of the cryopreserved ovarian tissue without need to undergo IVF $[2,9,13]$. The disadvantages of this procedure are need for surgical procedures for removal of ovarian tissue and transplant, it incurs more expenses, risk of reimplantation of malignant tissue and uncertainty regarding the longevity of function of the ovarian tissue after autotransplant [1].

After the first live birth following orthotopic ovarian autotransplant in 2004, the procedure is widely used and 60 live births have been reported worldwide so far in sterile cancer survivors using this method $[2,3,5,11]$. The main purpose of ovarian cryopreservation and autotransplant is restoration of fertility but the procedure also helps in restoration of physiological hormonal function of the ovaries thereby helping to improve the quality of life $[1,2]$. After chemotherapy and radiotherapy, $80-90 \%$ of women encounter premature ovarian failure which is associated with distressful symptoms for the patient. Conventionally, these menopausal symptoms are treated by hormone replacement therapy which is associated with side effects and moreover some patients are not even suitable for hormone replacement therapy. However, restoration of ovarian function by ovarian tissue transplant is more physiological in the sense that it makes use of the abundant endogenous store of naturally available ovarian follicles which would otherwise undergo atresia [1]. After orthotopic transplant of cryopreserved ovarian tissue, ovarian hormonal function typically returns very quickly in 4 - 5 months, the average time required for follicular growth and maturation as evidenced by resumption of menses and rise in estradiol levels [7, 8, 10]. Restoration of the normal hormonal function may help to alleviate menopausal symptoms like hot flushes, loss of libido, improve memory and sleep function and may also have a beneficial effect on osteoporosis and cardiovascular health, but all these beneficial effects are yet to be scientifically proven $[1,4,11]$.

It is not unreasonable to offer ovarian tissue cryopreservation to young women with aim of restoring the hormonal function alone even if they are not keen for fertility. The most important fact the patient needs to be aware is the issue of uncertainty of longevity of the ovarian tissue function following transplant as there are no tests for estimation of duration of its function and varies in individuals as it depends on a multitude of factors like age at which the tissue was retrieved, ovarian reserve, amount of tissue retrieved and the method used for transplant $[1,5,7]$. The longevity of the ovarian function varies in different studies, in one study it lasted for at least 1 year in $70 \%$ of cases, lasted longer in a few other studies and there is even a report of maintenance of ovarian activity for as long as 11 years after the ovarian transplant $[1-3,11,13]$. Apart from the risks involved in surgical procedure, another 
major safety concern includes the risk of reintroduction of malignancy, the magnitude of which is unknown for different cancers and some patients with cancers like hematological cancers and those with high risk of metastasis in ovarian tissue are not suited for the procedure $[10,13]$. Risk of reintroduction of malignancy can be minimized if ovarian tissue is harvested after one round of chemotherapy and a sample of the cryopreserved ovarian tissue is subjected to histological analysis to rule out any evidence of malignancy before transplant [12].

Careful selection of cases, individually tailored advice, multidisciplinary involvement, adequate psychological counselling, detailed discussion regarding the benefits, risks, cost, limitations and alternatives of the procedure and close monitoring are prerequisites to optimize outcome in the procedure [4]. Finally, whether this procedure can be utilized to postpone menopause in patients without medical indications or in women who request social freezing to postpone childbearing is highly questionable and controversial as risks may outweigh benefits in these circumstances [1].

\section{Conclusion}

The option of cryopreservation and autotransplant of ovarian tissue can be considered not only for fertility preservation in young women undergoing gonadotoxic treatment but also for women who wish to have restoration of endocrine and hormonal function for various health benefits. More cases with long-term follow-up are needed before we can recommend this treatment for restoration of hormonal function alone. Moreover, the cut off age below which the procedure should be offered is debatable and may vary according to policies and statistics in different institutions.

\section{Grant Support}

None.

\section{References}

1. von Wolff M, Stute P. Cryopreservation and transplantation of ovarian tissue exclusively to postpone menopause: technically possible but endocrinologically doubtful. Reprod Biomed Online. 2015;31(6):718-721.
2. Stoop D, Cobo A, Silber S. Fertility preservation for agerelated fertility decline. Lancet. 2014;384(9950):13111319.

3. Donnez J, Dolmans MM. Ovarian cortex transplantation: 60 reported live births brings the success and worldwide expansion of the technique towards routine clinical practice. J Assist Reprod Genet. 2015;32(8):1167-1170.

4. Patrizio P, Caplan AL. Forever young? The ethical challenges of using ovarian tissue transplants to treat menopause. Reprod Biomed Online. 2015;31(2):132-133.

5. Andersen CY, Silber SJ, Bergholdt SH, Jorgensen JS, Ernst E. Long-term duration of function of ovarian tissue transplants: case reports. Reprod Biomed Online. 2012;25(2):128-132.

6. Schmidt KT, Rosendahl M, Ernst E, Loft A, Andersen AN, Dueholm M, Ottosen C, et al. Autotransplantation of cryopreserved ovarian tissue in 12 women with chemotherapy-induced premature ovarian failure: the Danish experience. Fertil Steril. 2011;95(2):695-701.

7. Donnez J, Dolmans MM, Pellicer A, Diaz-Garcia C, Sanchez Serrano M, Schmidt KT, Ernst E, et al. Restoration of ovarian activity and pregnancy after transplantation of cryopreserved ovarian tissue: a review of 60 cases of reimplantation. Fertil Steril. 2013;99(6):1503-1513.

8. Donnez J, Martinez-Madrid B, Jadoul P, Van Langendonckt A, Demylle D, Dolmans MM. Ovarian tissue cryopreservation and transplantation: a review. Hum Reprod Update. 2006;12(5):519-535.

9. Donnez J, Jadoul P, Squifflet J, Van Langendonckt A, Donnez O, Van Eyck AS, Marinescu C, et al. Ovarian tissue cryopreservation and transplantation in cancer patients. Best Pract Res Clin Obstet Gynaecol. 2010;24(1):87-100.

10. Grynberg M, Poulain M, Sebag-Peyrelevade S, le Parco S, Fanchin R, Frydman N. Ovarian tissue and follicle transplantation as an option for fertility preservation. Fertil Steril. 2012;97(6):1260-1268.

11. Silber S, Pineda J, Lenahan K, DeRosa M, Melnick J. Fresh and cryopreserved ovary transplantation and resting follicle recruitment. Reprod Biomed Online. 2015;30(6):643-650.

12. Kolp LA, Hubayter Z. Autotransplantation of cryopreserved ovarian tissue: a procedure with promise, risks, and a need for a registry. Fertil Steril. 2011;95(6):18791886.

13. Ovarian tissue cryopreservation: a committee opinion. Fertil Steril. 2014;101(5):1237-1243. 